\title{
Transfer Learning Based System for Melanoma Type Detection
}

\author{
Rashmi Patil*, Sreepathi Bellary \\ Computer Science and Engineering, RYMEC, VTU, Bellary 583275, India
}

Corresponding Author Email: rashmipatil@rymec.in

https://doi.org/10.18280/ria.350203

Received: 20 January 2021

Accepted: 11 March 2021

\section{Keywords:}

melanoma, skin cancer, type of melanoma, transfer learning, border detection

\begin{abstract}
Skin disease is found in different sorts, for example, basal, squamous cell carcinoma and melanoma among which melanoma is one that is very difficult to predict. Finding melanoma at an early stage is crucial. Melanomas come in many forms and may display none of the typical warning signs. Early detection can vastly increase chances for the cure. Computer vision can assume significant part in Medical Image Diagnosis and it has been demonstrated by numerous existing frameworks. Here, we represent computer aided strategy for identification of type of melanoma utilizing the transfer learning techniques. The proposed model utilized pre-trained and transfer learning model to image net. The proposed model successfully classified three melanoma types, namely, Nodular melanoma, Lentigo maligna melanoma and Superficial spreading melanoma. Additionally, exact identification of irregular borders from melanoma skin lesions is clinically significant. A main challenge is deciding the specific lesion border. For resolving the issue, we have executed another technique to identify the border of an affected or cancerous area. A dataset consists of 2475 dermoscopic images to train and test algorithms. Performance of a proposed system gave very good results as far as sensitivity, accuracy, specificity, recall, fscore, and precision. At last, we have compared performance of different transfer learning techniques.
\end{abstract}

\section{INTRODUCTION}

Melanoma is a sort of skin malignancy that emerge when color delivering cells called melanocytes change and start to isolate wildly. A set of cancer cells which produce on the body and extinguish tissue is called a cancerous tumor. There is some other name is also present for melanoma such as malignant and cutaneous melanoma.

Melanomas can occur anyplace on the skin, especially some parts are more at danger compare to others. It is destined to influence the chest and back portion of men. And the legs are the most common site in women. It may also happen on a face, in an eye and other areas of the body.

Melanocytes produce melanin that produces hair, skin and eyes their shading. The skin is a body's biggest organ and spreads on the full body. It shields against harm caused by germs, sweltering temperatures and the sun. The skin maintains internal heat level, eliminates by products from the body through perspiration and gives the feeling of touch. It additionally helps make nutrient $\mathrm{D}$. It gathers and shapes moles on the skin. They show up as knocks which are a normally earthy colored. The vast majority has a couple of moles and are non-dangerous. However, sometimes, changes to melanocytes can cause melanoma skin malignant growth. An adjustment in the shading, size or state of a mole is normally the principal indication of melanoma skin malignant growth. Melanoma can likewise begin in different pieces of the body where melanocytes are found, yet these kinds of melanoma are discovered uncommon.

Superficial spreading melanoma, Lentigo maligna melanoma, Acral lentiginous melanoma, Nodular melanoma, and Amelanotic melanoma are some different types of melanoma [1]. Nodular melanoma grows downwards into a skin, Superficial spreading melanoma started growing outwards of the skin which is a top layer of skin. While Lentigo maligna melanoma raises in the outward surface layer of skin and is an initial form of melanoma known as melanoma in situ, acral lentiginous melanoma found on hand palms and feet soles also it can grow under the nails and Amelanotic melanoma consist of red or skin color melanin.

- Superficial spreading melanoma is a widely recognized kind of melanoma skin cancer. It begins to grow outward and also spread over the surface of the skin. In any case, it can likewise begin to develop down into the skin. Mostly, it is thin with a lopsided fringe. It changes in shading and may have various shades of red, grey, blue, earthy colored, dark, and white. In some cases, it begins from a mole that is as of now on the skin. It normally creates on the focal aspect of the body (trunk), legs and arms. It will in general occur on the back of gents and the legs of ladies.

- Lentigo maligna melanoma mostly found in aged people. It normally shows up as a huge, flat tan or an earthy colored fix with a lopsided outskirt. It will in general get more obscure as it develops and has numerous shades of earthy colored or dark. It regularly begins from an in-situ tumor called lentigo maligna, which is an early type of development just in the top or external layer of the skin. It typically becomes outward over the outside of the skin for a long time before it begins to develop down into the skin. It for the most part creates on regions of skin that are consistently 
presented to the sun without security, for example, the face, ears, and arms.

- Nodular melanoma is also common kinds of melanoma skin cancer. It develops down into a skin and spreads more rapidly than different sorts of melanoma skin malignancy. The development might be molded like a mushroom with a stem or tail. It is typically dark, yet in some cases can be a similar tone as skin, red or pink. It mainly creates on the chest, face, or back. It very wells may be found on zones of skin not presented to the sun.

All types of melanoma require quick attention, as they are more successfully treated when caught in their early stages. If it is diagnosed at a beginning phase, then it is treatable and it helps to save life of person. The stage of cancer decides kind of treatment suitable.

Further, paper is arranged as follows: existing approaches are specified in 2nd section; system architecture, methodology and algorithms used are explained in 3rd section; dataset description, experimental setup, evaluation parameters and results analysis are discussed in the 4th section, and at last, the conclusion is mentioned.

\section{LITERATURE SURVEY}

Melanoma is known as deadliest skin cancer because it causes person death. When it is diagnosed at an early stage, then it is recoverable so it is vital to classify them in stages, but it is a very complicated task to identify and classify them into stages. Here Patil and Bellary [2] presented two methods to categorize stages of melanoma. In a first method, classifier classifies melanoma lesions into stage 1 or 2 and in a second method, it classifies melanoma lesions in stage 1,2 or 3 . The authors utilized convolutional neural network with loss function Similarity Measure for Text Processing.

Menzies' method discussed in paper [3] is based on a checklist of eleven features. In this method, a total of eleven features' absence or presence is investigated. It distinguishes benign lesions from melanoma by two negative and nine positive feature sets. The negative set includes only two features that are symmetry and single color, while the positive set includes nine features: an existence of blue-white veil, pseudo pods, peripheral black dots, multiple brown dots, radial streaming, multiple blue or gray dots, scar-like pigmentation, multiple colors, and a broad pigment network. An existence of at least one feature from the positive feature's list and absence of both features from a negative features list are necessary to diagnose a skin lesion as malignant melanoma.

The Seven-Point Checklist method discussed in the research [4], that uses the scoring technique as in ABCDE rule. There are three major and four minor evaluation criteria. Major criteria are the existence of atypical vascular pattern(s), a grayblue area(s), and an atypical pigment network(s) with the score of " 2 ." For instance, if all these criteria exist in a lesion it is scored as 6. There are also minor criteria. These are the existence of radial streaming, regression pattern, irregular dots and globule and irregular diffuse pigmentation in a skin lesion with the score of " 1 " for each. In order to make a melanoma diagnosis, the minimum total score of three is required.

Jaworek-Korjakowska et al. [5] presented a framework to detect melanoma cancer stage. Author mainly focused on melanoma thickness for that they utilized the convolutional neural network (CNN) with transfer learning VGG19 model. The prediction of melanoma thickness is completely based on
CNN and VGG19 model.

Gong et al. [6] presented a decision fusion method that depends on This predominantly illuminates a speculation problem of CNN. StyleGANs are trained utilizing the ISIC 2019 database, also it upgrades exactness of categorization.

Wang et al. [7] proposed bi-directional dermoscopic include learning plan that extracts features. Image parsing capacity is enhanced by controlling component engendering. A multiscale steady decision fusion approach is additionally presented to improve consistency and dependability. Skin lesion segmentation done by utilizing the Bi-directional dermoscopic feature learning and multi-scale consistent decision fusion.

Wei et al. [8] presented segmentation and detection of skin disease from dermoscopic pictures. It depends on a lightweight deep learning network. The strategy can extricate discriminatory lesion features and furthermore enhances distinguishing a proof execution of the model.

Zhang et al. [9] presented system that utilize enhanced whale optimization algorithm to enhance $\mathrm{CNN}$. An enhanced algorithm is utilized for ideal choice of loads and inclinations in a network to lessen an error of network result and ideal result. They studied detection of skin cancer using optimized $\mathrm{CNN}$ and $\mathrm{AI}$.

Pattern analysis is another method that is used to analyze melanocytic sores and to separate benign melanocytic from melanoma. A pattern analysis method is used to identify specific patterns of skin lesions that can be either global or local. Some global patterns are globular, reticular, cobblestone, homogeneous, multicomponent, starburst, and nonspecific, which referred to benign melanocytic lesions. Local patterns are dots, vascular structures, globules, a pigment network, streaks, moles, blue-whitish veil, blotches, hypopigmentation, and regression structures which are also refer to benign melanocytic lesions [10].

Celebi et al. [11] extricated shape, texture and shading and took care of these element vectors to classifier with the end goal that they were positioned utilizing feature selection algorithms to decide deal subset size. The methodology outcome is a sensitivity of $93.33 \%$ and a specificity of $92.34 \%$ utilizing 564 pictures.

Dreiseitl et al. [12] examined robustness of artificial neural networks (ANN), support vector machines (SVM), k-nearest neighbors, logistic regression, and decision trees on characterizing regular nevi, melanoma and dysplastic nevi. They tended to three characterization issues: a dichotomous issue of isolating normal nevi from melanoma and dysplastic nevi, and a trichotomous issue of really isolating every one of these classes. They revealed that on the two cases logistic regression, SVMs and ANNs indicated a similar presentation, though k-nearest neighbor and decision trees performed more regrettable.

Rubegni et al. [13] extracted texture features, other than shading and shape highlights. ANN based methodology arrived at the specificity $93 \%$ and sensitivity of $96 \%$ on a database of 558 images consisting of 217 melanoma cancer.

Iyatomi et al. [14] presented a web-based framework which utilizes feature vector comprising shape, a surface, and shading highlights. They accomplished sensitivity and specificity of $86 \%$ utilizing 1200 dermoscopy images. Local approaches have additionally been as of late functional for skin sore order.

If it is diagnosed in early stages, skin cancer is $90 \%$ treatable compared to $50 \%$ in late stages [15]. With the development of noninvasive and high-resolution imaging techniques, the 
accuracy of in-situ diagnosis of skin cancers or skin lesions has increased. Especially, the lower diagnostic accuracy for melanoma is the major reason for over treatment (caused by false positive diagnosis) or under treatment (caused by false negative diagnosis). False positive diagnosis is the major contributor of excessive treatment cost increases due to leading to excise an unnecessarily high number of benign lesions for biopsy and pathological examination.

ABCDE method was introduced in 1994 by Nachbar et al. [16]. ABCDE stands for asymmetry, border, color, diameter, and evolving by time which are five dermoscopic criteria for semi-quantitative assessment of skin lesions. Melanomas are typically asymmetric with jagged edges and bigger than $6 \mathrm{~mm}$. They usually have mixed colors along with changing size, color, shape, and bleeding. These criteria (except E) have their possible scores based on the look of the skin lesion. These scores are multiplied by associated weight factors to gives a total dermoscopy score (TDS).

\section{METHODOLOGY}

\subsection{Our contribution}

The contributions of proposed work:

(1) We added more filters to layers, by this, changed the design of the ImageNet which upgrade features and reduce noise. We have used four transfer learning techniques with Adam and RMSprop optimiser and crossentropy and mean square error loss function. By this, the detection of a type of melanoma cancer has been accomplished.

(2) Lesion border plays a crucial role during surgery. We have proposed and designed a method to automatically identify the border of cancerous skin lesion.

(3) The results of different transfer learning algorithms are compared.

\subsection{System architecture}

The architecture of a system is shown in Figure 1. Dataset is of melanoma cancer and in this proposed system we are detecting type of melanoma cancer. A dataset consists of 2475 dermoscopic images to train and test algorithms. Different types of melanoma cancer that our system detects are Superficial spreading melanoma, Nodular melanoma and Lentigo maligna melanoma. Detailed dataset description is given in Table 1. The input dataset is given to different transfer learning algorithms, including MobileNet, ResNet50, VGG16 and VGG19 for training the system. The test dataset is passed to the system for evaluation and graphs will be generated for obtained results. The system will evaluate and check the type of melanoma cancer (Superficial spreading melanoma, Nodular melanoma, Lentigo maligna melanoma). The proposed system further identifies the border of affected lesion, which in turn will help for diagnosis and surgery.

\subsection{Malignant classification with transfer learning}

Transfer learning is one of popular techniques on computer vision and deep learning field to transfer knowledge from one domain to another. Transfer learning allows users to utilize pre-trained weights from another domain in case of limited computational power.

\subsubsection{Transfer-learning-MobileNet}

We shall be using Mobilenet as it is lightweight in its framework. It utilizes depth wise distinct convolutions which fundamentally implies it plays out a solitary convolution on each shading channel as opposed to joining every one of the three and flattening it. This has the impact of sifting the information channels. It is likewise extremely low support along these lines performing very well with fast.

\subsubsection{Transfer-learning-ResNet50}

The Residual Network, or ResNet was technologically advanced by researchers at Microsoft and defined in the 2015 [17]. The model needs color images to have size $224 \times 224$. Resnet50 folder has 2 pre-trained weights files xyz_tf_kernels.h5 and xyz_tf_kernels_NOTOP.h5. The xyz_tf_kernels.h5 weights is useful for pure prediction of test image, and this prediction will rely completely on ResNet50 pre-trained weights, i.e., it does not expect any training from our side. Our intention in this kernel is transfer learning by using ResNet50 pre-trained weights except its TOP layer, i.e., xyz_tf_kernels_NOTOP.h5 weights. Use these weights as an initial weight for training new layer using train images.

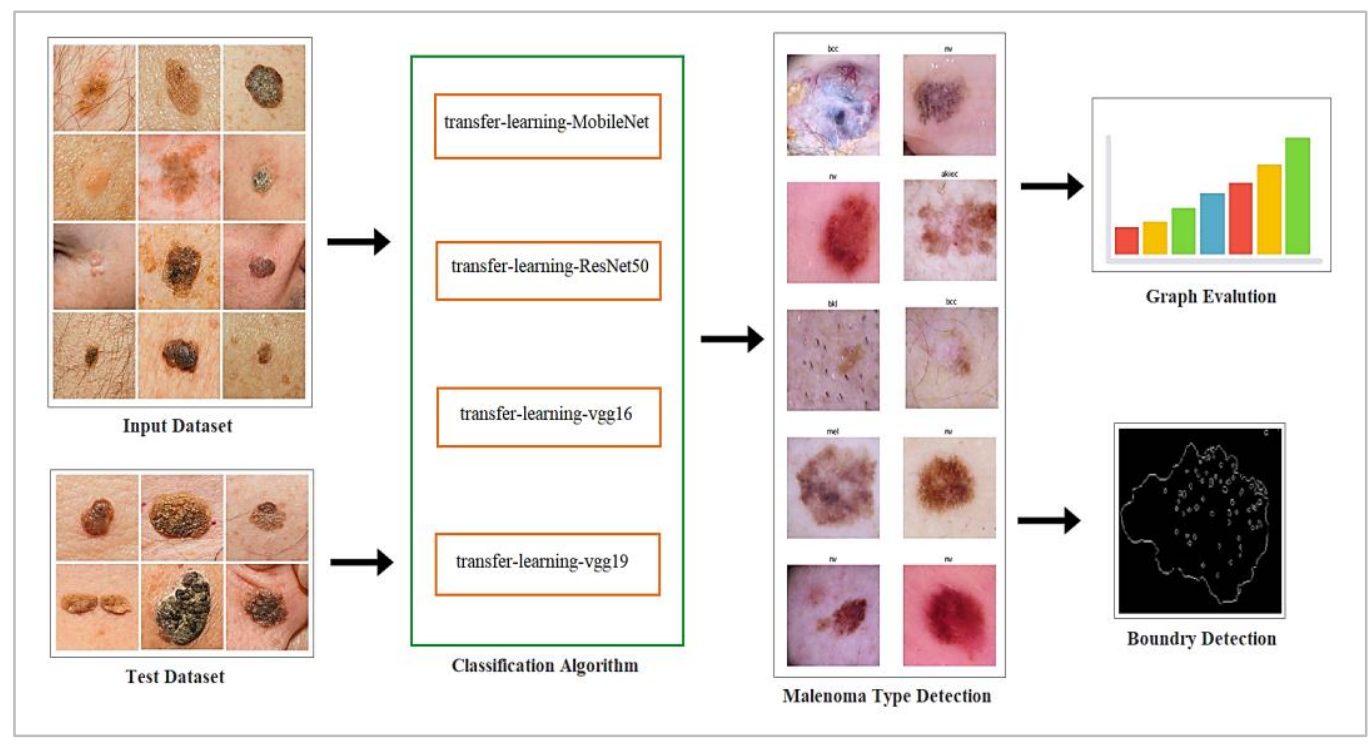

Figure 1. System architecture 


\subsubsection{Transfer-learning-VGG16}

It is a moderately direct model to utilize and a straightforward model framework to comprehend. We utilized VGG16 model to categorize new pictures. To begin with, the image requires to stacked and resized to $224 \times 224$, this is required by the model, and pixel esteems scaled in a route expected by model. Model works on a variety of tests, hence components of a stacked picture should be extended by 1 , for one picture with $224 \times 224$ pixels and three channels. Next, the model can be stacked and an expectation made. Last layers of VGG16 model are completely associated layers before yield layer. These layers will give an unpredictable arrangement of highlights to portray a given information picture and may give helpful information when preparing another model for image order or related PC vision task.

\subsubsection{Transfer-learning-VGG19}

A VGG19 architecture is basic one. On the off chance that you comprehend the fundamental CNN model, you will in a split-second notification that VGG19 seems to be comparative.

\subsection{Border detection}

Specifically, border recognition is a basic computer vision problem attributable to a wide scope of lesion tone, sizes, skin texture types, and shapes. An exact and precise identification feature data which is pertinent for a high dependable conclusion, for example, lesion area asymmetry and irregularity. Presented a basic technique for a skin sore division which can play out an exact border identification. Working out the border of melanoma lesions is a significant advance for dermatologists, who need a precise method of arriving at the right clinical finding. The proposed system detects a border of affected lesion. The border detection system is shown in Figure 2. Figure 3 shows dermoscopic image of a lesion, inverted image and border detected image.

Image Border Detection

1. Input Image

2. Blur image

3. Convert to Binary

4. Detect the contours in the image

5. Draw all the contours

6. invert image

7. merge image or border detection

Input Image: The input image is dermoscopic image of affected skin lesion

Blur Image: Blurring of image is accomplished by convolving the image with a low-pass filter kernel. This is helpful to remove noise. High frequency content such as edges, noise is removed from the image resulting in edges being blurred when this is filter is applied. cv2.blur() method is used to blur an image using the normalized box filter. In our work $75 \%$ blur image of original image is done. The function smooths an image using the kernel which is represented as:

$$
K=\frac{1}{\text { ksize.width } * \text { ksize. height }}\left[\begin{array}{ccc}
1 & \cdots & 1 \\
\vdots & \ddots & \vdots \\
1 & \cdots & 1
\end{array}\right]
$$

where, ksize.width is image width and ksize.height is image height.

Counters: Contours are essentially a curve that connects all of the continuous points (along the boundary) that are the same colour or brightness. The contours are a valuable method for object identification and recognition as well as shape analysis.
It can also be used to draw any form as long as the boundary points are known. For better accuracy we have perform binary image conversion also, before finding contours, image thresholding is performed. For contours detection function from OpenCV find Contours() is used. As the first of three return parameters, the function no longer modifies the source image but instead returns a modified image.

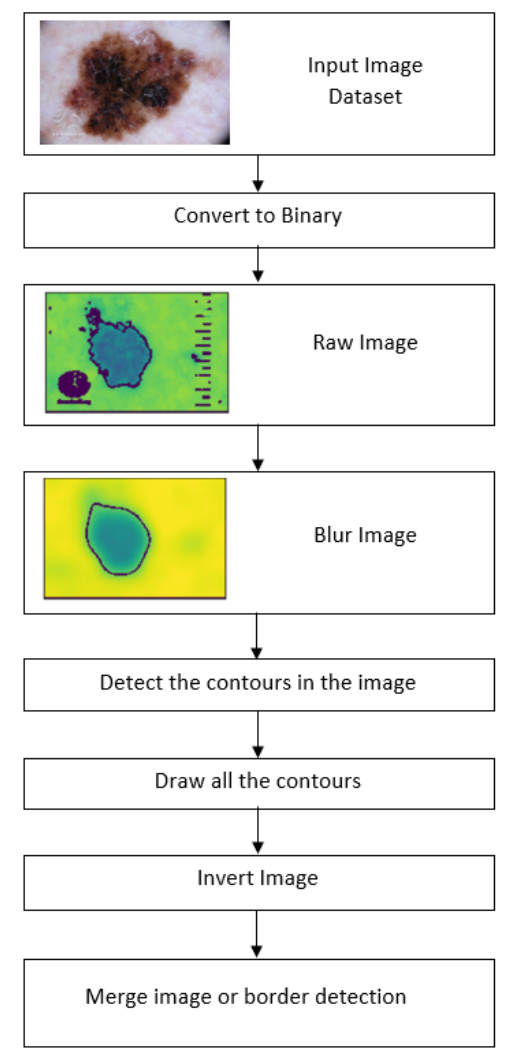

Figure 2. Border detection system

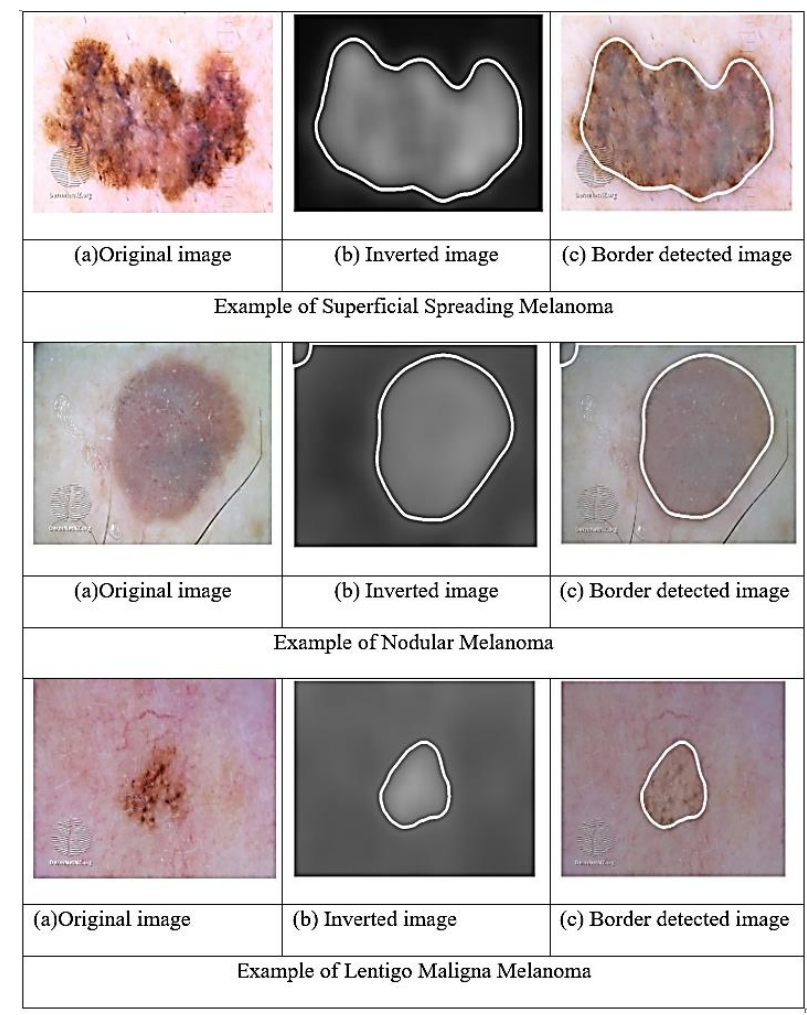

Figure 3. Types of malenoma and border detection 
Invert Image: Color Inversion (Image Negative) is the method of inverting pixel values of an image. Image inversion does not depend on the color mode of the image, i.e., inversion works on channel level. When inversion is used on a multi color image (RGB, CMYK etc.) then each channel is treated separately, and the final result if formed by calibrating the results of all the channels.

The method used for getting the inverse of an image is subtraction of the maximum value/intensity of a pixel by the value of current pixel. The resultant value is guided by the formula:

$$
I M G_{\text {Inverted }}=I M G_{\text {Max }}-I M G_{(x, y)}
$$

where, $I M G_{\text {Inverted }}$ is inverted image,

$I M G_{M a x}$ is maximum intensity value,

$I M G_{(x, y)}$ is intensity value of current pixel.

Merge Image for Border Detection: Finally, we have merged the inverted image with the original image to get exact border of the affected lesion.

\section{RESULTS AND ANALYSIS}

\subsection{Dataset escription}

The dataset is used from https://dermnetnz.org/ [18]. To avoid overfitting, we have used a data augmentation method. It is a method to artificially create new training data from existing training data. A zoom augmentation is used to augment the images. It randomly zooms the image in and either adds new pixel values around the image or interpolates pixel values respectively.

Table 1 shows detailed about dataset: Type 1: Superficial spreading melanoma, Type 2: Nodular melanoma and Type 3: Lentigo maligna melanoma.

\subsection{Experimental setup}

Windows 10 OS equipped with a Core i7 processor and 8 GB DDRAM with a GeForce MX150 NVIDIA graphics card

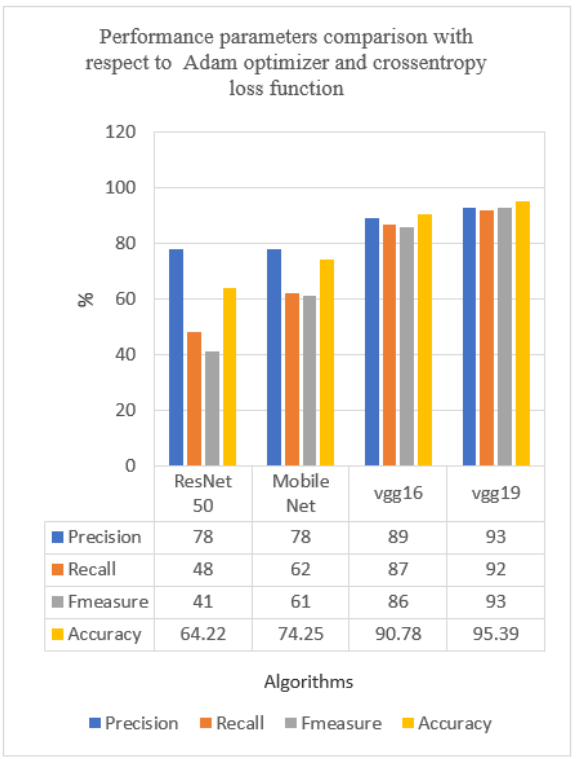

Figure 4. Performance parameters comparison with respect to Adam optimizer and cross entropy loss function and 500 GB SSD was used to perform the experiments. An Anaconda IDE was utilized to execute a program. Training epochs number is 30 , and batch size is 32 ; the initial learning rate was 0.001 .

Table 1. Dataset information

\begin{tabular}{cccc}
\hline & Type 1 & Type 2 & Type 3 \\
\hline Training Dataset & 656 & 530 & 549 \\
\hline Testing Dataset & 280 & 226 & 234 \\
\hline Total & 936 & 756 & 783 \\
\hline
\end{tabular}

\subsection{Evaluation metrics}

The confusion matrix is used for the evaluation model. It is represented by a table which describes the performance of a classification model on a set of test data in machine learning. Proposed work performance is assessed utilizing accuracy, recall, sensitivity, f score, specificity, and precision. Measures are calculated from:

$$
\begin{gathered}
\text { Accuracy }=\frac{T_{p}+T_{n}}{T_{p}+T_{n}+F_{p}+F_{n}} \\
\text { Sensitivity }=\frac{T_{p}}{T_{p}+T_{n}} \\
\text { Specificity }=\frac{T_{n}}{F_{p}+T_{n}} \\
\text { Precision }=\frac{T_{p}}{T_{p}+F_{p}} \\
\text { Recall }=\frac{T_{p}}{T_{p}+T_{n}}
\end{gathered}
$$

$$
F 1=2 X \frac{\text { Precision } x \text { Recall }}{\text { Precision }+ \text { Recall }}
$$

where, $T_{p}, F_{p}, F_{n}, T_{n}$ represents to true positive, true negative, false negative, and false positive.

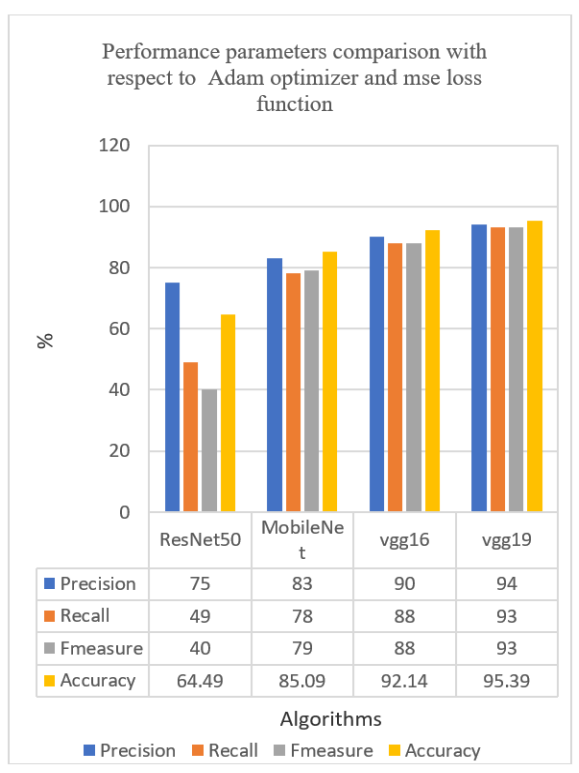

Figure 5. Performance parameters comparison with respect to Adam optimizer and mse loss function 
Performance Parameters comparison with respect to RMS optimizer and crossentropy loss function

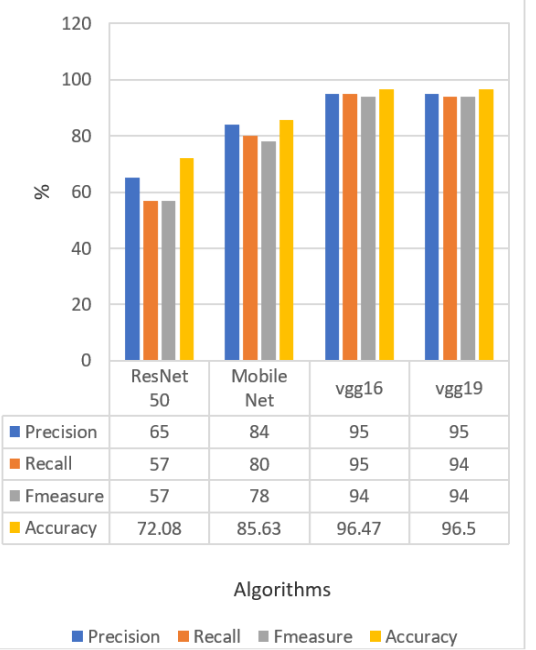

Figure 6. Performance parameters comparison with respect to RMS optimizer and cross entropy loss function

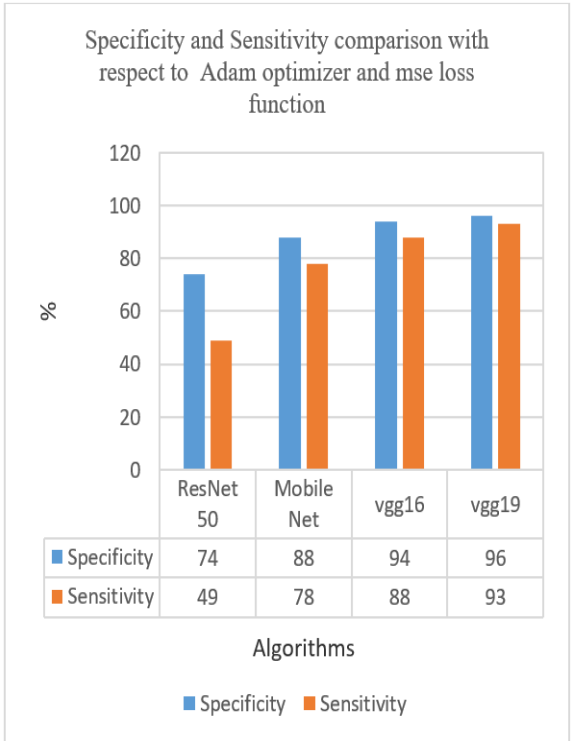

Figure 9. Specificity and sensitivity comparison graph with respect to Adam optimizer and mse loss function

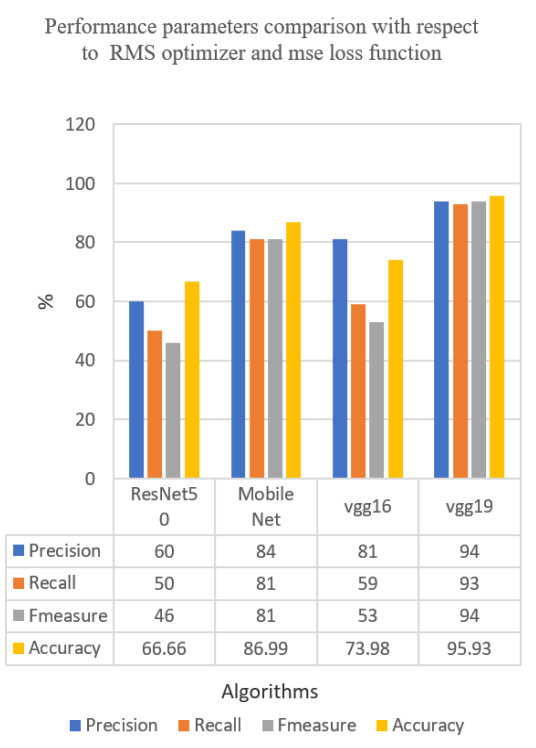

Figure 7. Performance parameters comparison with respect to RMS optimizer and mse loss function

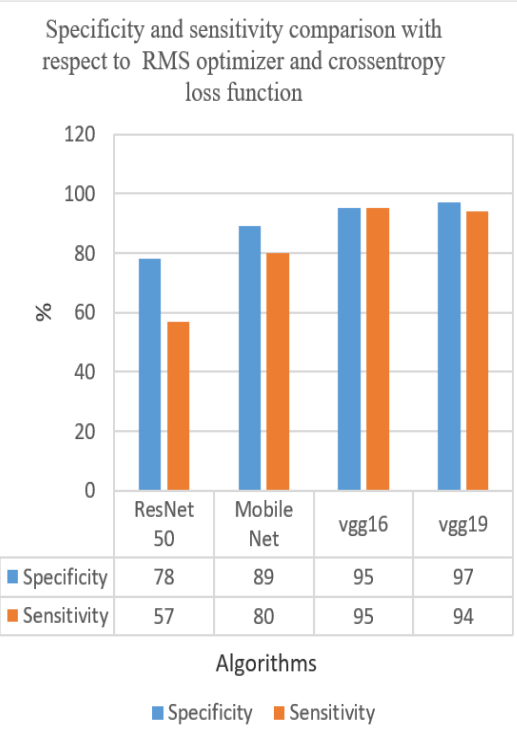

Figure 10. Specificity and sensitivity comparison with respect to RMS optimizer and cross entropy loss function
Specificity and sensitivity comparison with respect to Adam optimizer and crossentropy loss function

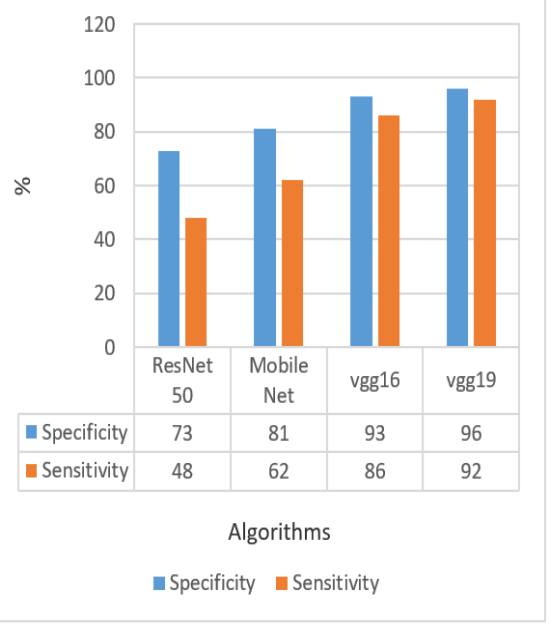

Figure 8. Specificity and sensitivity comparison with respect to Adam optimizer and cross entropy loss function

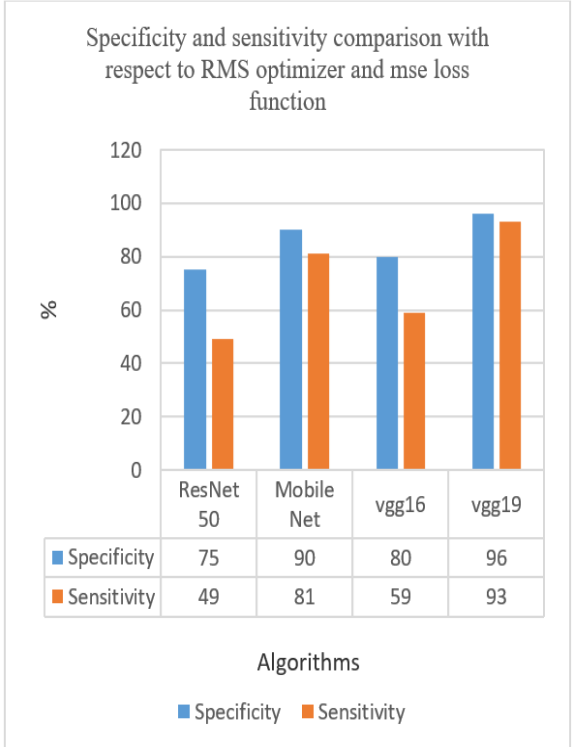

Figure 11. Specificity and sensitivity comparison with respect to RMS optimizer and mse loss function

\subsection{Analysis}

Different transfer learning algorithms are trained and tested on dataset [18-20]. Dataset information is given in Table 1. Transfer learning algorithm such as ResNet50, MobileNet, VGG16 and VGG 19 are used to detect the type of melanoma. The results obtained by each algorithm is compared by others. The performance parameter comparison is accomplished using Adam optimizer and crossentropy loss function, Adam optimizer and mean square error (mse) loss function, RMSprop optimizer and crossentropy loss function, RMSprop optimizer and mean square error loss function. Performance evaluation parameters, including, accuracy, sensitivity, specificity, precision, recall, and fscore are calculated and represented in comparison graphs. In Figure 4 to Figure 7 shows the comparison of results of four transfer learning methods with combination of different optimisers (Adam and RMSprop) and loss functions (mse and crossentropy). Comparatively VGG19 gives better results in terms of accuracy, f measure, precision and recall. In Figure 8 to Figure 11 shows the comparison of sensitivity and specificity transfer learning methods with different optimisers and loss functions. Again, VGG19 results better to other transfer learning techniques. Figure 12 shows training and validation comparison graphs. 

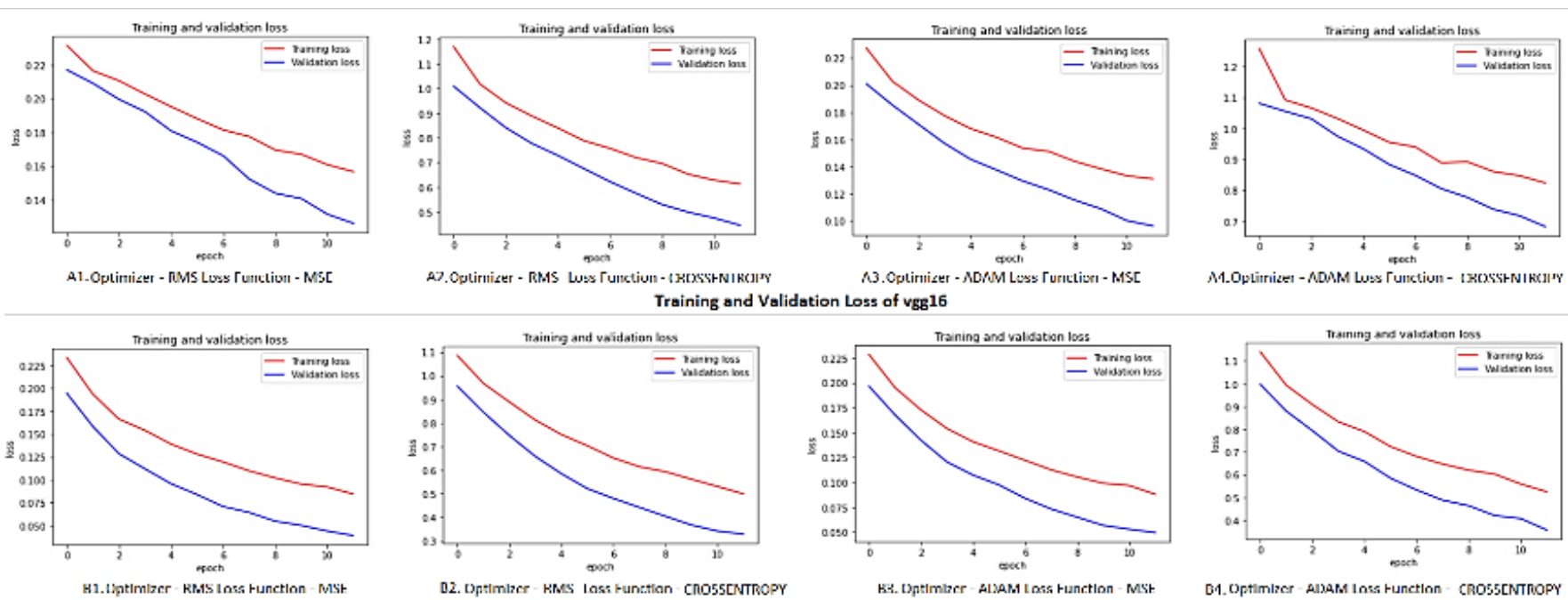

2. Dptimlecr- RMS Ioss Function CnO5SCNTROPY

Ba. Dptimiter - Aliasa Loss tunction - MSt

Q4. Optimizer -anam Iosa tunction - CROS5CNTROPY

Training and Validation Loss of vgg19
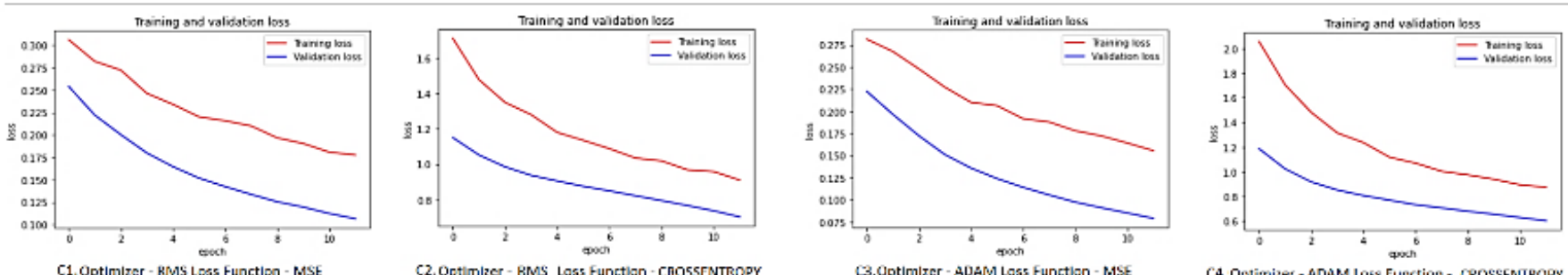

Training and Validation Loss of MOBILE NET

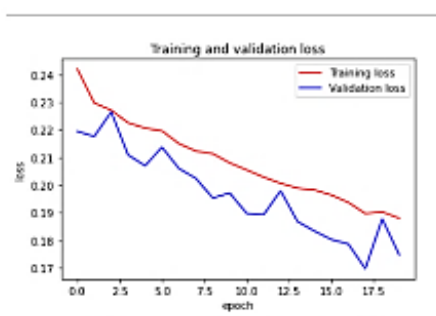

D1. Optirnizer - RMAS Loss Function - MSE

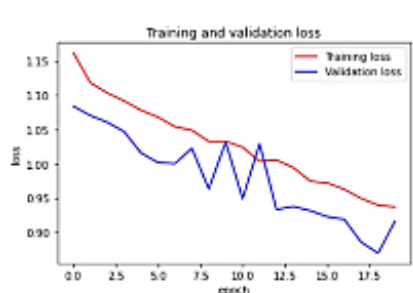

D2. Optimizer - RMS LOSS Function - CROSSENTROPY

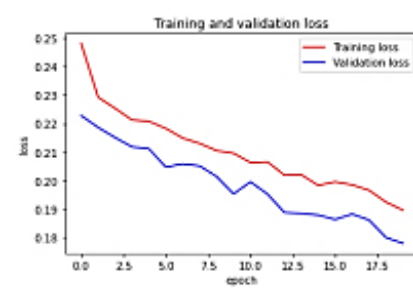

D3. Optimizer - ADAM Loss Function - MSE

TralnIng and Valldatlon Loss of ResNet50

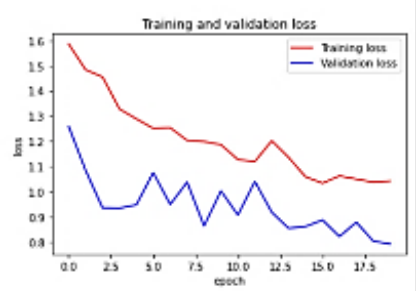

D4.Optinizer - ADMM Loss Function - CROSSENTROPY

Figure 12. Training and Validation Loss comparison graphs of A1) RMS optimizer and mse loss function of VGG16 A2) RMS optimizer and crossentropy loss function of VGG16 A3) Adam optimizer and mse loss function of VGG16 A4) Adam optimizer and crossentropy loss function of VGG16 B1) RMSprop optimizer and mse loss function of VGG19 B2) RMS optimizer and crossentropy loss function of VGG19 B3) Adam optimizer and mse loss function of VGG19 B4) Adam optimizer and crossentropy loss function of VGG19 C1) RMS optimizer and mse loss function of MobileNet C2) RMS optimizer and crossentropy loss function of MobileNet C3) Adam optimizer and mse loss function of MobileNet C4) Adam optimizer and crossentropy loss function of MobileNet D1) RMS optimizer and mse loss function of ResNet50 D2) RMS optimizer and crossentropy loss function of ResNet50 D3) Adam optimizer and mse loss function of ResNet50 D4) Adam optimizer and crossentropy loss function of ResNet50

\section{CONCLUSION}

One of the principle undertakings of current dermatology is the identification of a type of melanoma cancer in its beginning phase. Due to this, there may be an increase in the survival rate. This can be accomplished by joining novel instruments with computer-aided diagnosis. A point of frameworks is to enhance sensitivity and specificity of a type of melanoma detection, also lessen unwanted biopsies. An affected lesion border is very significant factor for assessment of melanoma skin lesion. The proposed system is evaluated using performance measures includes, sensitivity, accuracy, specificity, precision, $f$ score and recall. From experimental results obtained, it is observed that VGG19 performs better compared to ResNet50, VGG16 and MobileNet in terms of sensitivity, accuracy, specificity, precision, f score and recall.
When transfer learning architecture layer's weights were finetuned, performance measures become higher than only finetuning with replaced layers. The framework targets at diminishing deep learning architecture complexity in identifying a type of melanoma. The main purpose of developing an effective system is to meet up with real time medical diagnosis task in diagnosing melanoma skin cancer. It can be decided from outcomes that proposed system can be efficiently utilized by physicians and also, patients to diagnose types of melanoma with more accuracy.

\section{REFERENCES}

[1] Type of melanoma skin cancer. https://www.cancer.ca/en/cancer-information/cancer- 
type/skin-melanoma/melanoma/types-of-

melanoma/?region=on, accessed on Jan. 10, 2021.

[2] Patil, R., Bellary, S. (2020). Machine learning approach in melanoma cancer stage detection. Journal of King Saud University-Computer and Information Sciences, 19. https://doi.org/10.1016/j.jksuci.2020.09.002

[3] Menzies Method from dermoscopedia. https://dermoscopedia.org/w/index.php?title=Menzies Method\&oldid=9988, accessed on Jan. 10, 2021.

[4] 7-Point Checklist. https://dermlite.com/pages/7-pointchecklist, accessed on Jan. 10, 2021.

[5] Jaworek-Korjakowska, J., Kleczek, P., Gorgon, M. (2019). Melanoma thickness prediction based on convolutional neural network with VGG-19 model transfer learning. 2019 IEEE/CVF Conference on Computer Vision and Pattern Recognition Workshops (CVPRW), Long Beach, CA, USA, pp. 2748-2756. http://dx.doi.org/10.1109/CVPRW.2019.00333

[6] Gong, A., Yao, X., Lin, W. (2020). Dermoscopy image classification based on StyleGANs and decision fusion. IEEE Access, 8: 70640-70650. https://doi.org/10.1109/ACCESS.2020.2986916

[7] Wang, X., Jiang, X., Ding, H., Liu, J. (2019). Bidirectional dermoscopic feature learning and multi-scale consistent decision fusion for skin lesion segmentation. IEEE Transactions on Image Processing, 29: 3039-3051. https://doi.org/10.1109/TIP.2019.2955297

[8] Wei, L., Ding, K., Hu, H. (2020). Automatic skin cancer detection in dermoscopy images based on ensemble lightweight deep learning network. IEEE Access, 8: 99633-99647. http://dx.doi.org/10.1109/ACCESS.2020.2997710.

[9] Zhang, N., Cai, Y.X., Wang, Y.Y., Tian, Y.T., Wang, X.L., Badami, B. (2020). Skin cancer diagnosis based on optimized convolutional neural network. Artificial Intelligence in Medicine, 102: 101756. https://doi.org/10.1016/j. artmed.2019.101756

[10] Masood, A. (2016). Developing improved algorithms for detection and analysis of skin cancer. Doctoral dissertation.

[11] Celebi, M.E., Kingravi, H.A., Uddin, B., Iyatomi, H., Aslandogan, Y.A., Stoecker, W.V., Moss, R.H. (2007).
A methodological approach to the classification of dermoscopy images. Computerized Medical Imaging and $\quad$ Graphics, 362-373. https://doi.org/10.1016/j.compmedimag.2007.01.003

[12] Dreiseitl, S., Ohno-Machado, L., Kittler, H., Vinterbo, S., Billhardt, H., Binder, M. (2001). A comparison of machine learning methods for the diagnosis of pigmented skin lesions. Journal of Biomedical Informatics, 34(1): 28-36. https://doi.org/10.1006/jbin.2001.1004

[13] Rubegni, P., Cevenini, G., Burroni, M., Perotti, R., Dell'Eva, G., Sbano, P. (2002). Automated diagnosis of pigmented skin lesions. International Journal of Cancer, 101(6): 576-580. https://doi.org/10.1002/ijc.10620

[14] Iyatomi, H., Oka, H., Celebi, M.E., Hashimoto, M., Hagiwara, M., Tanaka, M., Ogawa, K. (2008). An improved internet-based melanoma screening system with dermatologist-like tumor area extraction algorithm. Computerized Medical Imaging and Graphics, 32(7): 566-579. https://doi.org/10.1016/j.compmedimag.2008.06.005

[15] DiChiara, T. (2010). Pictures of Moles and Melanoma skin cancer-Learn o tell the difference with pictures.

[16] Nachbar, F., Stolz, W., Merkle, T., Cognetta, A.B., Vogt, T., Landthaler, M. (1994). The ABCD rule of dermatoscopy: High prospective value in the diagnosis of doubtful melanocytic skin lesions. Journal of the American Academy of Dermatology, 30(4): 551-559. https://doi.org/10.1016/S0190-9622(94)70061-3

[17] He, K., Zhang, X., Ren, S., Sun, J. (2016). Deep residual learning for image recognition. 2016 IEEE Conference on Computer Vision and Pattern Recognition (CVPR), Las Vegas, NV, USA, pp. 770-778. https://doi.org/10.1109/CVPR.2016.90

[18] Superficial spreading melanoma images. https://dermnetnz.org/topics/superficial-spreadingmelanoma-images/, accessed on Dec. 01, 2020.

[19] Lentigo maligna and lentigo maligna melanoma images. https://dermnetnz.org/topics/lentigo-malignamelanoma-images/, accessed on Dec. 01, 2020.

[20] Nodular melanoma images. https://dermnetnz.org/topics/nodular-melanoma-images/, accessed on Dec. 01, 2020. 